\title{
Distribution of populations in excited states of electrodeless discharge lamp of Rb atoms
}

\author{
TAO ZhiMing ${ }^{1,2}$, WANG YanFei ${ }^{1}$, HONG YeLong ${ }^{1}$, WANG DongYing ${ }^{1}$, ZHANG ShengNan ${ }^{1}$, \\ ZHUANG Wei $^{1 *}$ \& CHEN JingBiao ${ }^{1}$ \\ ${ }^{1}$ State Key Laboratory of Advanced Optical Communication System and Network, Institute of Quantum Electronics, School of Electronics \\ Engineering \& Computer Science, Peking University, Beijing 100871, China; \\ ${ }^{2}$ Department of Physics, Bijie University, Bijie 551700, China
}

Received September 29, 2012; accepted January 5, 2013

The intensity of fluorescence spectral lines of $\mathrm{Rb}$ atoms in the region of 350-1110 nm is measured in eletrodeless discharge lamp. The population ratio between the excited states is calculated according to the spontaneous transition probabilities with rate equations. At the same time, the population density of energy level is also obtained. The results provide the potential applications of electrodeless discharge lamp in atomic filter and optical frequency reference at higher excited states without a pumping laser.

electrodeless discharge lamp, population ratio, population density

Citation: Tao Z M, Wang Y F, Hong Y L, et al. Distribution of populations in excited states of electrodeless discharge lamp of Rb atoms. Chin Sci Bull, 2013, 58: 1876-1881, doi: 10.1007/s11434-013-5789-Z

The electrodeless discharge lamp of $\mathrm{Rb}$ atoms containing $\mathrm{Rb}$ vapor and buffer gas is an important component of the microwave $\mathrm{Rb}$ atomic clock [1,2]. Nowadays, they play a central role in applied physics and analytical chemistry [3,4], metrology such as the $\mathrm{Rb}$ atoms frequency standard and alkali magnetometer [5]. The reason why the lamp has been used for optical pumping [6] instead of lasers in these devices is mainly that the electrodeless discharge lamp can yield fluorescence with stable frequency and higher signal-to-noise ratios [7]. The fluorescence of the electrodeless discharge lamp can also be used as a pumping light source for active optical clock [8,9], multi-threshold second-order phase transition [10], and Faraday anomalous dispersion optical filter [11].

In recent years our group has begun a series of experiments to investigate the population distribution of some main excited states of the electrodeless discharge lamp [12]. In this work, we discuss the experiments and results investigating the distribution of excited states from visible light

*Corresponding author (email: wzhuang@pku.edu.cn) to near-infrared in the region of $350-1110 \mathrm{~nm}$.

\section{Experimental setup}

As shown in Figure 1, the bulb in lamp is a cylindrical glass cell with a length of $3 \mathrm{~cm}$ and a diameter of $3 \mathrm{~cm}$. It contains natural $\mathrm{Rb}$ atoms and $\mathrm{Xe}$ gas at $2.7 \times 10^{2} \mathrm{~Pa}$, the bulb is supplied with $178 \mathrm{MHz}$ rf (radio frequency) power and can be heated from room temperature to $200^{\circ} \mathrm{C}$. The lamp is designed to output light from both sides of the bulb, so a laser beam could pass through the lamp. The USB2000+ spectrometer produced by Ocean Optics Company in USA with a resolution of $1.5 \mathrm{~nm}$ is used to measure the fluorescence spectra.

When heating voltage is set to $10 \mathrm{~V}$, about 20 min later, the temperature of lamp is heated from room temperature to $120^{\circ} \mathrm{C}$. At the moment, $\mathrm{rf}$ is switched on. When rf voltage reaches $8 \mathrm{~V}$, the lamp lights. At this time, the lamp is working at the red mode.

After electrons and ions of the lamp containing $\mathrm{Rb}$ atoms 


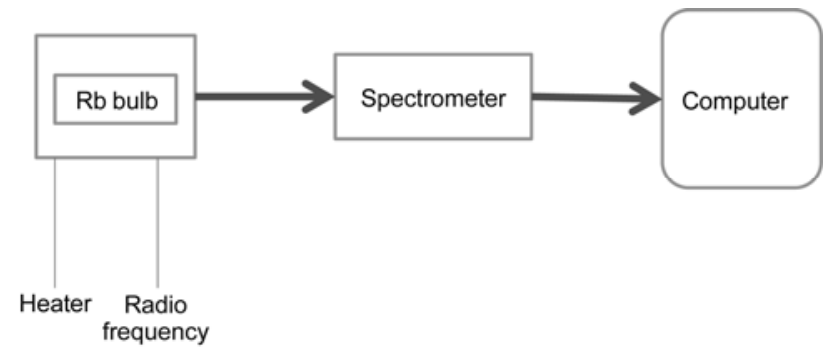

Figure 1 The experimental scheme for spectrum research of Rb electrodeless discharge lamp.

and $\mathrm{Xe}$ atoms are accelerated by the high frequency electric field, their energy increases [1]. These electrons and ions with high energy are collided with Xe atoms. More electrons and ions are produced. These electrons and ions with high energy excite Xe atoms to high level. When Xe atoms transit from high level to low level by spontaneous radiation, they can release the fluorescence. At that time, the spectra of Xe atoms appear using the spectrometer. If these Xe atoms in excited states are collided with $\mathrm{Rb}$ atoms, energy can be transferred from $\mathrm{Xe}$ atoms in excited states to $\mathrm{Rb}$ atoms. $\mathrm{Xe}$ atoms go back to ground state through no radiation transition when they excite $\mathrm{Rb}$ atoms from ground state to excited states. Similarly, when $\mathrm{Rb}$ atoms transit from high level to low level by spontaneous radiation, the spectra of $\mathrm{Rb}$ atoms appear.

As there are $\mathrm{Xe}$ atoms in the $\mathrm{Rb}$ bulb, the spectra measured are divided into two parts. One is the spectra of $\mathrm{Rb}$ atoms which is just the subject investigated in this work.
The other is the spectra of Xe atoms which is not the subject investigated here. Therefore, in this paper, we do not consider the fluorescence spectra of Xe atoms.

\section{Results}

\subsection{Intensity of spectral lines of $\mathbf{R b}$ atoms}

The lamp can operate in three spectral modes. They are the ring mode, the red mode and the weak mode respectively [12]. When changing the rf power from low to high, the weak, the red and the ring modes appear in sequence. No matter which mode the lamp is operating in, the population ratio between the excited states of $\mathrm{Rb}$ atoms is almost constant. For simplicity, we do not consider the working mode when the intensity of fluorescence spectral lines of Rb atoms is measured.

Figure 1 shows the block diagram of our experimental arrangement, and Figure 2 shows the energy diagram of the transitions related to the spectral signal. The energy levels of $\mathrm{Rb}$ atoms that we need to measure are located in the region of 350-1110 $\mathrm{nm}$. We pay special attention to transition between the excited states on the blue-green band, which can be used to realize submarine communication, underwater communication. In order to facilitate reference, we draw other energy levels of $\mathrm{Rb}$ atoms.

The intensity of different spectral lines of $\mathrm{Rb}$ atoms varies widely, of which $780 \mathrm{~nm}$ is the strongest, $795 \mathrm{~nm}$ is ranked second and $520 \mathrm{~nm}$ is the weakest in our measurements. As the intensity of spectral lines measured by spectrometer is

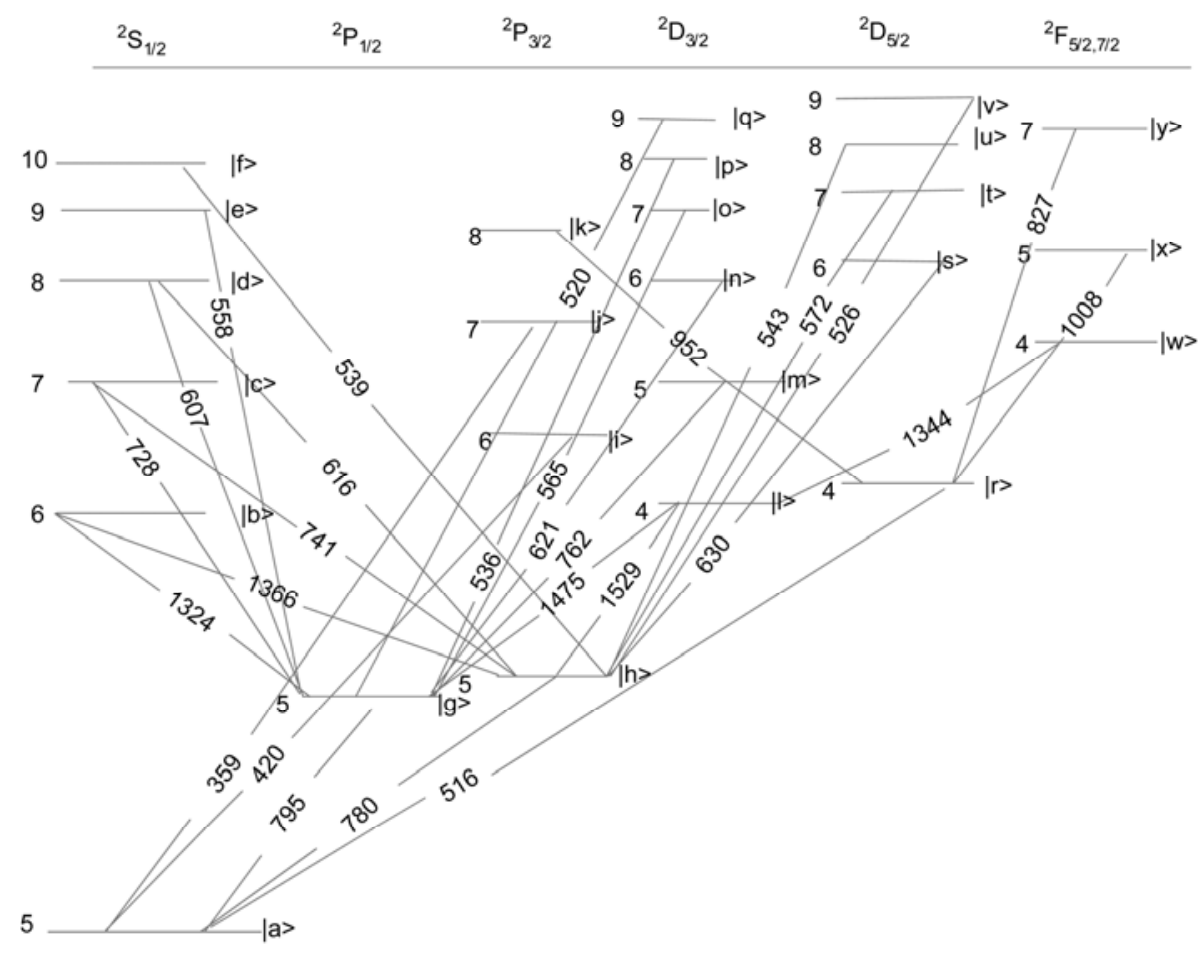

Figure 2 The energy level diagram of the transitions. 
limited, when the intensity of $780 \mathrm{~nm}$ is close to saturation, that of $519 \mathrm{~nm}$ is still very weak and almost invisible. So it is necessary to measure the intensity of spectral lines group by group. For the same lines, the intensity of spectral lines varies with distance between the spectrometer and rubidium lamp. In order to compare the intensity of spectral lines, they are converted in accordance with the intensity value of $780 \mathrm{~nm}$. The following several spectra are measured according to different precision.

When the distance between the probe of spectrometer and $\mathrm{Rb}$ lamp is set appropriately, the relative intensities of $780,795,883 \mathrm{~nm}$ can be detected (Figure 3). Their relative intensities in arbitrary units are 61709, 51131, 5006, respectively. As shown in the Figure 3, the relative intensity of $780 \mathrm{~nm}$ is the strongest, $795 \mathrm{~nm}$ is ranked second and 883 $\mathrm{nm}$ is ranked third. $420 \mathrm{~nm}$ is next to $883 \mathrm{~nm}$, which is not assigned to the spectrum line of $\mathrm{Rb}$ atoms and is used for the reference intensity.

Figure 4 shows that the relative intensity of the $420 \mathrm{~nm}$ can be calculated according to the relative intensity of 883 $\mathrm{nm}$. The relative intensity of $420 \mathrm{~nm}$ is 1385 .

As shown in the Figure 5, the relative intensities of 572, 616, 621, 630, 728, 741 and $762 \mathrm{~nm}$ are 181, 153, 316, 550, $236,357,657$, respectively according to the relative intensity of $420 \mathrm{~nm}$.

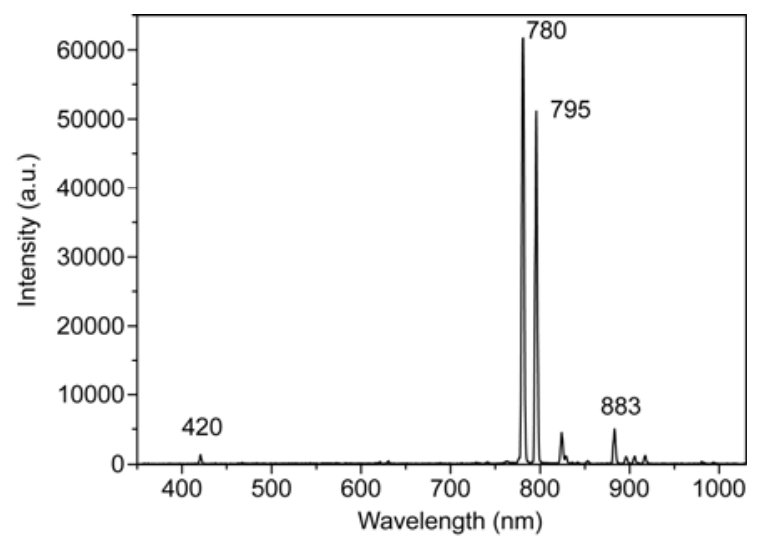

Figure 3 The intensity of $420,780,795,883 \mathrm{~nm}$.

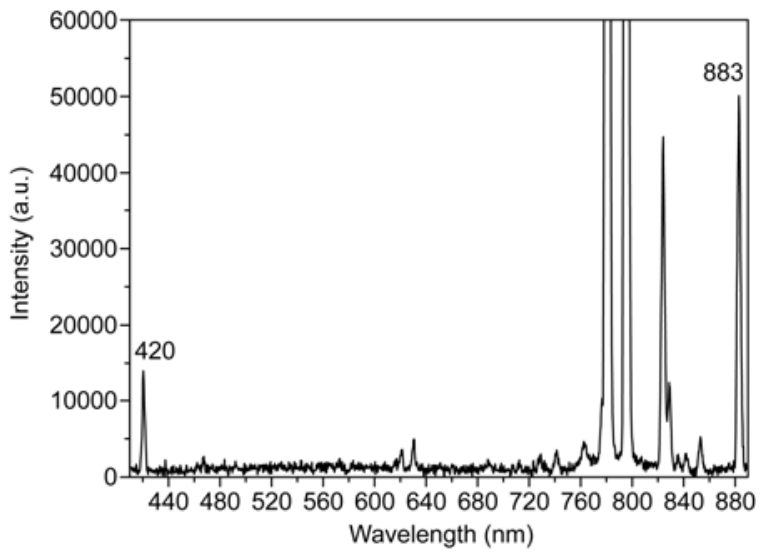

Figure 4 The intensity of $420,883 \mathrm{~nm}$

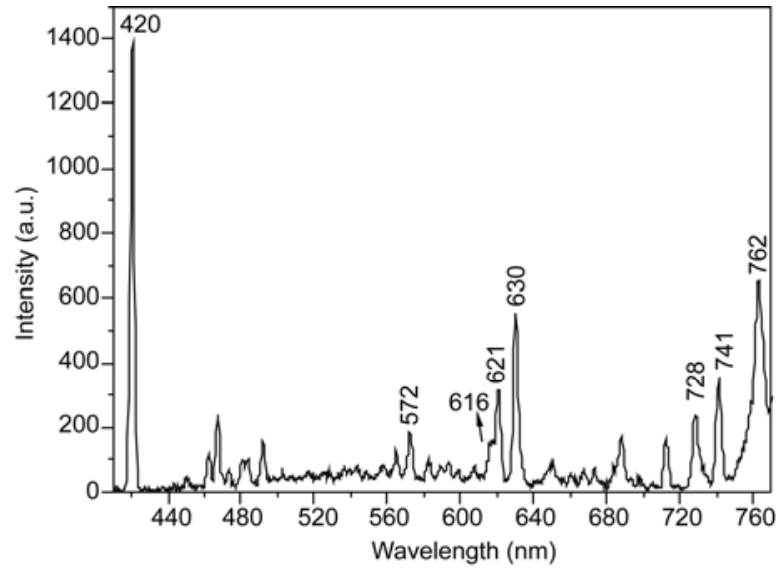

Figure 5 The intensity of 420, 572, 616, 621, 630, 728, 741, $762 \mathrm{~nm}$.

The relative intensities of the other spectra lines can be measured in the same way. Figure 6 shows that the relative intensities of $827,836 \mathrm{~nm}$ can be obtained by the relative intensity of $762 \mathrm{~nm}$. Their relative intensities are 2724,326 , respectively.

As shown in Figure 7, the relative intensities of $516 \mathrm{~nm}$ can be obtained by the relative intensity of $616 \mathrm{~nm}$. The relative intensities of 516, 520, 526, 536, 539, 543, 558, 565 and $607 \mathrm{~nm}$ are $31,14,29,51,31,77,57,144$ and 88, respectively. Likewise, Figure 8 shows that the relative intensity of $359 \mathrm{~nm}$ can be obtained by the relative intensity of $516 \mathrm{~nm}$. The relative intensities of 952, $1008 \mathrm{~nm}$ can be obtained by the relative intensity of $836 \mathrm{~nm}$ (Figure 9). The relative intensities of 359, 952 and $1008 \mathrm{~nm}$ are 61, 62 and 88 , respectively.

Table 1 lists all the spectra measured. In column 1, the spectral intensities are listed with different wavelengths, and they are listed by their relative intensities in column 3 of Table 1.

\subsection{Population ratio between excited states calculated by rate equations}

The corresponding reduced oscillator strengths are calculated

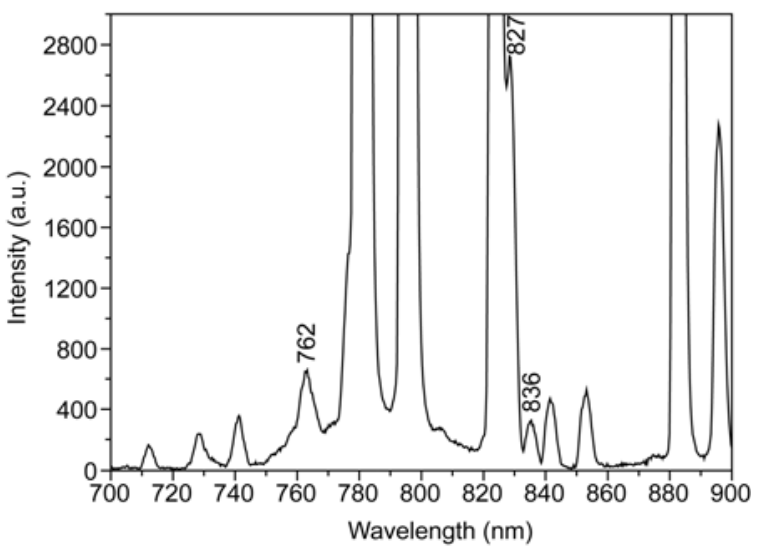

Figure 6 The intensity of $762,827,836 \mathrm{~nm}$. 


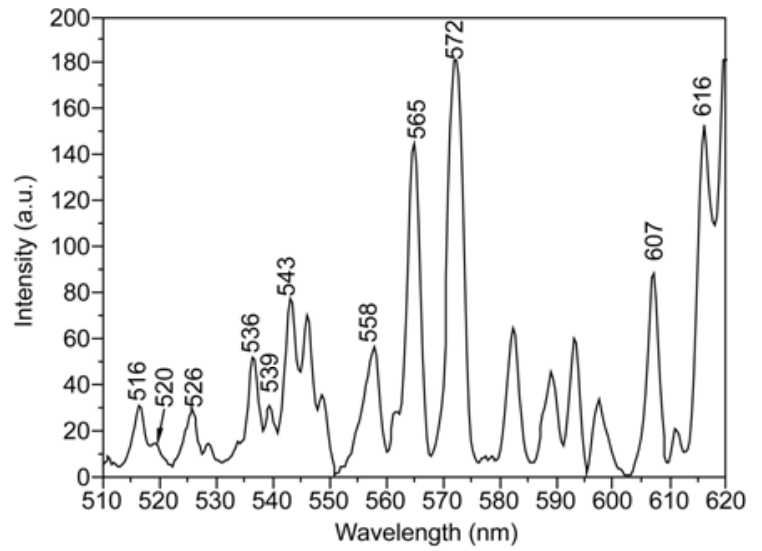

Figure 7 The intensity of 516, 520, 526, 536, 539, 543, 558, 565, 572, $607,616 \mathrm{~nm}$

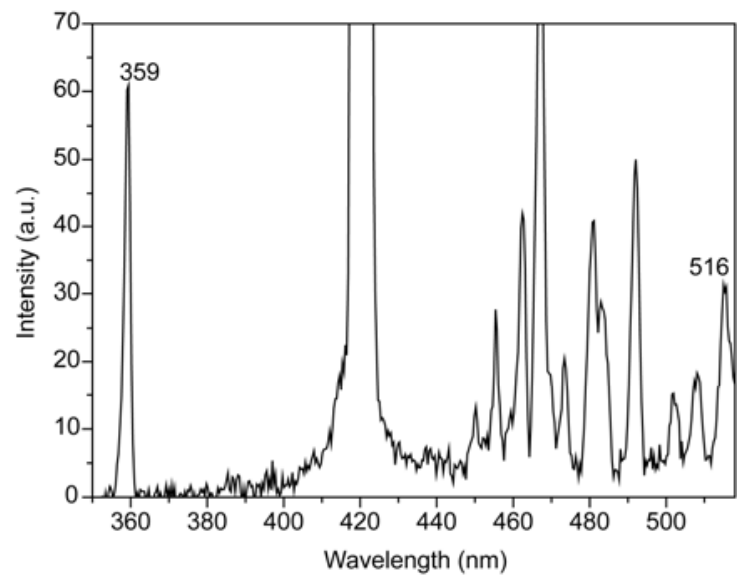

Figure 8 The intensity of $359,516 \mathrm{~nm}$

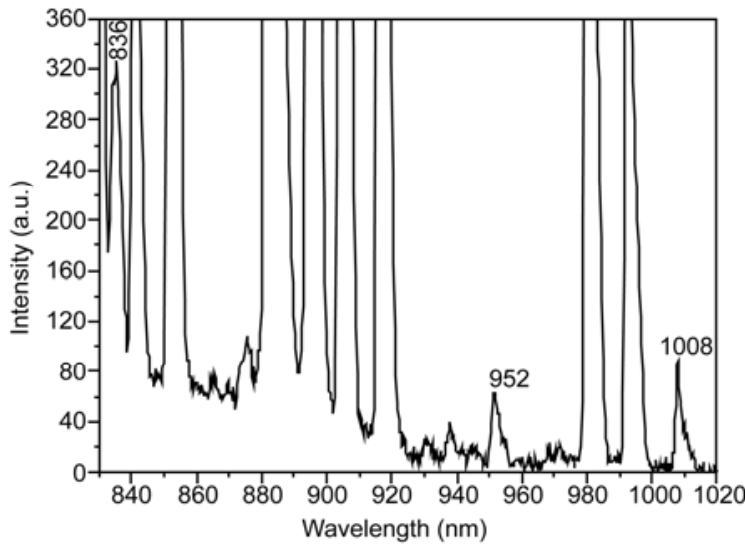

Figure 9 The intensity of 836, 952, $1008 \mathrm{~nm}$.

using the formula [15]:

$$
f_{\mathrm{ab}}=-\frac{30.3756}{\left(2 j_{\mathrm{a}}+1\right) \lambda}|<a\|D\| b>|^{2} \text {. }
$$

The Einstein $A$ coefficients $A_{\mathrm{ab}}$ are calculated using the formula [15]:

$$
A_{\mathrm{ab}}=\frac{2.02613 \times 10^{15}}{\lambda^{3}} \frac{\left.|<a\|D\| b\rangle\right|^{2}}{2 j_{\mathrm{a}}+1} .
$$

Therefore we have $A_{\mathrm{ab}}=\frac{6.67 \times 10^{13}}{\lambda^{2}} f_{\mathrm{ab}}$.

The value of $f_{607}$ and $f_{616}$ is 0.0063 and 0.0124 [15]. The corresponding values of $A_{607}$ and $A_{616}$ are 1.14 and 2.17, respectively.

\begin{tabular}{|c|c|c|c|c|c|}
\hline$\lambda(\mathrm{nm})$ & $I$ (a.u.) & \multirow{2}{*}{ Transition level } & $\lambda(\mathrm{nm})$ & $I$ (a.u.) & \multirow{2}{*}{ Transition level } \\
\hline \multicolumn{2}{|c|}{ Wavelength in order } & & \multicolumn{2}{|c|}{ Intensity in order } & \\
\hline 359 & 61 & $7^{2} \mathrm{P}_{3 / 2} \rightarrow 5^{2} \mathrm{~S}_{1 / 2}$ & 780 & 61709 & $5^{2} \mathrm{P}_{3 / 2} \rightarrow 5^{2} \mathrm{~S}_{1 / 2}$ \\
\hline 420 & 1385 & $6^{2} \mathrm{P}_{3 / 2} \rightarrow 5^{2} \mathrm{~S}_{1 / 2}$ & 795 & 51131 & $5^{2} \mathrm{P}_{1 / 2} \rightarrow 5^{2} \mathrm{~S}_{1 / 2}$ \\
\hline 516 & 31 & $4^{2} \mathrm{D}_{5 / 2} \rightarrow 5^{2} \mathrm{~S}_{1 / 2}$ & 827 & 2724 & $7^{2} \mathrm{~F}_{7 / 2} \rightarrow 4^{2} \mathrm{D}_{5 / 2}$ \\
\hline 520 & 14 & $9^{2} \mathrm{D}_{3 / 2} \rightarrow 5^{2} \mathrm{P}_{1 / 2}$ & 420 & 1385 & $6^{2} \mathrm{P}_{3 / 2} \rightarrow 5^{2} \mathrm{~S}_{1 / 2}$ \\
\hline 526 & 29 & $9^{2} \mathrm{D}_{5 / 2} \rightarrow 5^{2} \mathrm{P}_{3 / 2}$ & 762 & 657 & $5^{2} \mathrm{D}_{3 / 2} \rightarrow 5^{2} \mathrm{P}_{1 / 2}$ \\
\hline 536 & 51 & $8^{2} \mathrm{D}_{3 / 2} \rightarrow 5^{2} \mathrm{P}_{1 / 2}$ & 630 & 550 & $6^{2} \mathrm{D}_{5 / 2} \rightarrow 5^{2} \mathrm{P}_{3 / 2}$ \\
\hline 539 & 31 & $10^{2} \mathrm{~S}_{1 / 2} \rightarrow 5^{2} \mathrm{P}_{3 / 2}$ & 741 & 357 & $7^{2} \mathrm{~S}_{1 / 2} \rightarrow 5^{2} \mathrm{P}_{3 / 2}$ \\
\hline 543 & 77 & $8^{2} \mathrm{D}_{5 / 2} \rightarrow 5^{2} \mathrm{P}_{3 / 2}$ & 621 & 316 & $6^{2} \mathrm{D}_{3 / 2} \rightarrow 5^{2} \mathrm{P}_{1 / 2}$ \\
\hline 558 & 57 & $9^{2} \mathrm{~S}_{1 / 2} \rightarrow 5^{2} \mathrm{P}_{1 / 2}$ & 728 & 236 & $7^{2} \mathrm{~S}_{1 / 2} \rightarrow 5^{2} \mathrm{P}_{1 / 2}$ \\
\hline 565 & 144 & $7^{2} \mathrm{D}_{3 / 2} \rightarrow 5^{2} \mathrm{P}_{1 / 2}$ & 572 & 181 & $7^{2} \mathrm{D}_{5 / 2} \rightarrow 5^{2} \mathrm{P}_{3 / 2}$ \\
\hline 572 & 181 & $7^{2} \mathrm{D}_{5 / 2} \rightarrow 5^{2} \mathrm{P}_{3 / 2}$ & 616 & 153 & $8^{2} \mathrm{~S}_{1 / 2} \rightarrow 5^{2} \mathrm{P}_{3 / 2}$ \\
\hline 607 & 88 & $8^{2} \mathrm{~S}_{1 / 2} \rightarrow 5^{2} \mathrm{P}_{1 / 2}$ & 565 & 144 & $7^{2} \mathrm{D}_{3 / 2} \rightarrow 5^{2} \mathrm{P}_{1 / 2}$ \\
\hline 616 & 153 & $8^{2} \mathrm{~S}_{1 / 2} \rightarrow 5^{2} \mathrm{P}_{3 / 2}$ & 607 & 88 & $8^{2} \mathrm{~S}_{1 / 2} \rightarrow 5^{2} \mathrm{P}_{1 / 2}$ \\
\hline 621 & 316 & $6^{2} \mathrm{D}_{3 / 2} \rightarrow 5^{2} \mathrm{P}_{1 / 2}$ & 1008 & 88 & $5^{2} \mathrm{~F}_{7 / 2} \rightarrow 4^{2} \mathrm{D}_{5 / 2}$ \\
\hline 728 & 236 & $7^{2} \mathrm{~S}_{1 / 2} \rightarrow 5^{2} \mathrm{P}_{1 / 2}$ & 952 & 62 & $8^{2} \mathrm{P}_{3 / 2} \rightarrow 4^{2} \mathrm{D}_{5 / 2}$ \\
\hline 741 & 357 & $7^{2} \mathrm{~S}_{1 / 2} \rightarrow 5^{2} \mathrm{P}_{3 / 2}$ & 359 & 61 & $7^{2} \mathrm{P}_{3 / 2} \rightarrow 5^{2} \mathrm{~S}_{1 / 2}$ \\
\hline 762 & 657 & $5^{2} \mathrm{D}_{3 / 2} \rightarrow 5^{2} \mathrm{P}_{1 / 2}$ & 558 & 57 & $9^{2} \mathrm{~S}_{1 / 2} \rightarrow 5^{2} \mathrm{P}_{1 / 2}$ \\
\hline 780 & 61709 & $5^{2} \mathrm{P}_{3 / 2} \rightarrow 5^{2} \mathrm{~S}_{1 / 2}$ & 536 & 51 & $8^{2} \mathrm{D}_{3 / 2} \rightarrow 5^{2} \mathrm{P}_{1 / 2}$ \\
\hline 795 & 51131 & $5^{2} \mathrm{P}_{1 / 2} \rightarrow 5^{2} \mathrm{~S}_{1 / 2}$ & 516 & 31 & $4^{2} \mathrm{D}_{5 / 2} \rightarrow 5^{2} \mathrm{~S}_{1 / 2}$ \\
\hline 827 & 2724 & $7^{2} \mathrm{~F}_{7 / 2} \rightarrow 4^{2} \mathrm{D}_{5 / 2}$ & 539 & 31 & $10^{2} \mathrm{~S}_{1 / 2} \rightarrow 5^{2} \mathrm{P}_{3 / 2}$ \\
\hline 952 & 62 & $8^{2} \mathrm{P}_{3 / 2} \rightarrow 4^{2} \mathrm{D}_{5 / 2}$ & 526 & 29 & $9^{2} \mathrm{D}_{5 / 2} \rightarrow 5^{2} \mathrm{P}_{3 / 2}$ \\
\hline 1008 & 88 & $5^{2} \mathrm{~F}_{7 / 2} \rightarrow 4^{2} \mathrm{D}_{5 / 2}$ & 520 & 14 & $9^{2} \mathrm{D}_{3 / 2} \rightarrow 5^{2} \mathrm{P}_{1 / 2}$ \\
\hline
\end{tabular}

Table 1 Spectral signal intensities and wavelengths 
The power $(P)$ of the transition signal between two energy levels can be expressed as $p_{\lambda}=n_{\mu} A_{\mu \eta} h v$ where $\lambda$ is the transition wavelength, $n_{\mu}$ is the atom density in the level numbered $\mu(\mu=\mathrm{a}, \mathrm{b}, \mathrm{c} \cdots), A_{\mu \eta}$ is the spontaneous transition probability between $\mu$ energy level and $\eta$ energy level, $v$ is the transition frequency and $h$ is the Plank constant. The transition studied can be expressed with rate equations clearly as

$$
\begin{aligned}
& p_{359}=n_{\mathrm{j}} A_{\mathrm{ja}} h v_{359}, \\
& p_{420}=n_{\mathrm{i}} A_{\mathrm{ia}} h v_{420}, \\
& p_{607}=n_{\mathrm{d}} A_{\mathrm{dg}} h v_{607}, \\
& p_{616}=n_{\mathrm{d}} A_{\mathrm{dh}} h v_{616}, \\
& p_{621}=n_{\mathrm{n}} A_{\mathrm{ng}} h v_{621}, \\
& p_{630}=n_{\mathrm{s}} A_{\mathrm{sh}} h v_{630}, \\
& p_{728}=n_{\mathrm{c}} A_{\mathrm{cg}} h v_{728}, \\
& p_{741}=n_{\mathrm{c}} A_{\mathrm{ch}} h v_{741}, \\
& p_{762}=n_{\mathrm{m}} A_{\mathrm{mg}} h v_{762}, \\
& p_{780}=n_{\mathrm{h}} A_{\mathrm{ha}} h v_{780}, \\
& p_{795}=n_{\mathrm{g}} A_{\mathrm{ga}} h v_{795} .
\end{aligned}
$$

The spontaneous transition probabilities $A_{\mu \eta}$ and the wavelengths involved in the calculation are listed in Table 2. The calculated results of $n_{\mu} / n_{\mathrm{h}}$ are shown in Table 3 .

The population density of the ${ }^{87} \mathrm{Rb}$ atoms in the $5 P_{3 / 2}$ level (|h>) is $0.3 \%\left(n_{\mathrm{h}}\right)$ according to [12]. Since we know $n_{\mathrm{h}}$, we can obtain the densities in all the excited states mentioned above.

The calculation results of $n_{\mu}$ are shown in Table 4. From the Table 4 , we know that the two values of $n_{\mathrm{d}}$ are 1.11 and 1.02 , respectively. The difference of between them is small and relative deviation is $10 \%$. For the same reason, the two values of $n_{\mathrm{c}}$ is also different according to $728 \mathrm{~nm}$ and 741 $\mathrm{nm}$. However, the difference between them with a relative

Table 2 Spontaneous transition probabilities and wavelengths

\begin{tabular}{ll}
\hline$A_{\mu \eta}\left(10^{6} \mathrm{~s}^{-1}\right)$ & \multicolumn{1}{c}{$\lambda(\mathrm{nm})$} \\
\hline$A_{\mathrm{ja}}=0.396$ & $\lambda_{359}=358.7050[13]$ \\
$A_{\mathrm{ia}}=1.77$ & $\lambda_{420}=420.1792[13]$ \\
$A_{\mathrm{dg}}=1.14$ & $\lambda_{607}=607.0754$ \\
$A_{\mathrm{dh}}=2.17$ & $\lambda_{616}=615.9626$ \\
$A_{\mathrm{ng}}=2.948$ & $\lambda_{621}=620.6309[13]$ \\
$A_{\mathrm{sh}}=3.712$ & $\lambda_{630}=629.88325[13]$ \\
$A_{\mathrm{cg}}=2.02$ & $\lambda_{728}=727.9996[14]$ \\
$A_{\mathrm{ch}}=3.87$ & $\lambda_{741}=740.8173[14]$ \\
$A_{\mathrm{mg}}=2.12$ & $\lambda_{762}=761.8933[14]$ \\
$A_{\mathrm{ha}}=38.1$ & $\lambda_{780}=780.0268[13]$ \\
$A_{\mathrm{ga}}=36.1$ & $\lambda_{795}=794.7603[13]$ \\
\hline
\end{tabular}

Table 3 The calculation results of $n_{\mu} / n_{\mathrm{h}}$

\begin{tabular}{ll}
\hline$n_{\mu} / n_{\mathrm{h}}$ & \multicolumn{1}{c}{ Value } \\
\hline$n_{\mathrm{j}} / n_{\mathrm{h}}$ & 0.043 \\
$n_{\mathrm{i}} / n_{\mathrm{h}}$ & 0.259 \\
& $0.037($ according to $607 \mathrm{~nm})$ \\
$n_{\mathrm{d}} / n_{\mathrm{h}}$ & $0.034($ according to $616 \mathrm{~nm})$ \\
$n_{\mathrm{n}} / n_{\mathrm{h}}$ & 0.053 \\
$n_{\mathrm{s}} / n_{\mathrm{h}}$ & 0.074 \\
$n_{\mathrm{c}} / n_{\mathrm{h}}$ & $0.067($ according to $728 \mathrm{~nm})$ \\
$n_{\mathrm{m}} / n_{\mathrm{h}}$ & $0.054($ according to $741 \mathrm{~nm})$ \\
$n_{\mathrm{g}} / n_{\mathrm{h}}$ & 0.187 \\
& 0.89 \\
\hline
\end{tabular}

Table 4 The calculation results of $n_{\mu}$

\begin{tabular}{ll}
\hline$n_{\mu}$ & Value $\left(10^{-4}\right)$ \\
\hline$n_{\mathrm{j}}$ & 1.29 \\
$n_{\mathrm{i}}$ & 7.77 \\
& 1.11 (according to $607 \mathrm{~nm})$ \\
$n_{\mathrm{d}}$ & $1.02($ according to $616 \mathrm{~nm})$ \\
& 1.59 \\
$n_{\mathrm{n}}$ & 2.22 \\
$n_{\mathrm{s}}$ & $2.01($ according to $728 \mathrm{~nm})$ \\
& $1.62($ according to $741 \mathrm{~nm})$ \\
$n_{\mathrm{c}}$ & 5.61 \\
$n_{\mathrm{m}}$ & 26.7 \\
$n_{\mathrm{g}}$ & $30[12]$ \\
$n_{\mathrm{h}}$ & $5.4[12]$ \\
$n_{\mathrm{b}}$ & $10.2[12]$ \\
$n_{\mathrm{l}}$ & $9.3[12]$ \\
$n_{\mathrm{r}}$ &
\end{tabular}

deviation of $20 \%$ is very big.

Because of the lack of other values of the spontaneous radiation probability, some values of $n_{\mu}$ cannot be obtained.

\section{Conclusion and discussion}

From the Table 4, we know some atoms density of energy level, which indicates that there are enough populations in excited states when the lamp is lit. The atoms density of $\mathrm{Rb}$ atoms in excited states varies widely, of which $n_{\mathrm{h}}$ is the biggest, $n_{\mathrm{g}}$ is ranked second and $n_{1}$ is ranked third. The next is $n_{\mathrm{r}}$ and $n_{\mathrm{i}}$ corresponding to, $516 \mathrm{~nm}, 420 \mathrm{~nm}$, respectively. The value of $n_{1}$ corresponds to $1475 \mathrm{~nm}$ or $1529 \mathrm{~nm}$ (Figure 2). Therefore, in the double resonance optical pumping experiment, the lamp may replace the pumping laser of $420 \mathrm{~nm}$, $516 \mathrm{~nm}, 1475 \mathrm{~nm}$ and $1529 \mathrm{~nm}$. As there is also the population in other excited states, the lamp may replace the pumping laser of other wavelengths. In conclusion, the lamp can be used in specific applications such as the frequency stabilization reference of the laser frequency standard and the 
excited atoms filters without a pump laser to get populations in the excited states $[16,17]$.

Though lamp can operate in three spectral modes, the population ratio between the excited states of $\mathrm{Rb}$ atoms is almost constant. Therefore we do not consider the working mode when the intensity of spectral lines of $\mathrm{Rb}$ atoms is measured.

The intensity of spectral lines of $\mathrm{Rb}$ atoms from visible light to near-infrared is measured. The population ratio between the excited states is calculated according to the spontaneous transition probabilities. At the same time, the population density of energy level is also obtained.

This work was supported by the National Natural Science Foundation of China (10874009 and 11074011).

1 Wang Y Q, Fu J S, Dong T Q, et al. Principle of Quantum Frequency Standard (in Chinese). Beijing: Science Press, 1986. 391

2 Riehle F. Frequency Standards. Weinheim: Wiley VCH, 2004. 248249

3 Lagalante A F. Atomic absorption spectroscopy: A tutorial review. Appl Spectrosc Rev, 1999, 34: 173-189

4 Bings N H, Bogaerts A, Broekaert J A C. Atomic spectroscopy. Anal Chem, 2004, 76: 3313-3336

5 Alexandrov E B, Bonch-Bruevich V A. Optically pumped atomic magnetometers after three decades. Opt Eng (Bellingham), 1992, 31: 711-717

6 Camparo J C, Klimcak C M. Generation of ion-acoustic waves in an inductively coupled, low-pressure discharge lamp. J Appl Phys, 2006, 99: 083306

7 Camparo J C. Conversion of laser phase noise to amplitude noise in an optically thick vapor. J Opt Soc Am B, 1998, 15: 1177-1186

8 Wang Y Q. Optical clocks based on stimulated emission radiation. Chin Sci Bull, 2009, 54: 347

9 Chen J B. Active optical clock. Chin Sci Bull, 2009, 54: 348-352

10 Zhuang W, Yu D S, Liu Z W, et al. Multi-threshold second-order phase transition in laser. Chin Sci Bull, 2011, 56: 3812-3816

11 Guo H, Dang A H, Han Y Q, et al. Faraday anomalous dispersion optical filter. Chin Sci Bull, 2010, 55: 527-533

12 Sun Q Q, Miao X Y, Sheng R W, et al. The near-infrared spectra and distribution of excited states of electrodeless discharge rubidium vapour lamps. Chin Phys B, 2012, 21: 033201

13 Sansonetti J E. Wavelengths, transition probabilities, and energy levels for the spectra of rubidium. J Phys Chem Ref Data, 2006, 35: 307-308

14 Heavens O S. Radiative transition probabilities of the lower excited states of the alkali metals. J Opt Soc Am, 1961, 51: 1058-1060

15 Safronova M S, Williams C J, Clark C W. Relativistic many-body calculations of electric-dipole matrix elements, lifetimes, and polarizabilities in rubidium. Phys Rev A, 2004, 69: 022509

16 Sun Q Q, Zhuang W, Liu Z W, et al. Electrodeless discharge vapo lamp based Faraday anomalous dispersion optical filter. Opt Lett, 2011, 36: 4611-4613

17 Miao X Y, Yin L F, Zhuang W, et al. Demonstration of an external-cavity diode laser system immune to current and temperature fluctuations. Rev Sci Instrum, 2011, 82: 086106

Open Access This article is distributed under the terms of the Creative Commons Attribution License which permits any use, distribution, and reproduction in any medium, provided the original author(s) and source are credited. 